\title{
Classical Solutions of SU(3) Yang-Mills Theory and Heavy Quark Phenomenology
}

\author{
O. Oliveira \\ Centro de Física Computacional, Departamento de Física, Universidade de Coimbra, 3004-516 \\ Coimbra, Portugal
}

\begin{abstract}
It is showed that potentials derived from classical solutions of the SU(3) Yang-Mills theory can provide confining potentials that reproduce the heavy quarkonium spectrum within the same level of precision as the Cornell potential.
\end{abstract}

In order to solve the classical Yang-Mills equations of motion, usually one writes an ansatz that simplifies the Euler-Lagrange equations and, hopefully, includes the relevant dynamical degrees of freedom. In [1] it was proposed a generalized Cho-FaddeevNiemi-Shabanov ansatz for the gluon field, where the gluon is given in terms of two vector fields, $\hat{A}_{\mu}$ and $Y_{\mu}^{a}$, and a covariant constant real scalar field $n^{a}$,

$$
A_{\mu}^{a}=n^{a} \hat{A}_{\mu}+\frac{3}{2 g} f_{a b c} n^{b} \partial_{\mu} n^{c}+Y_{\mu}^{a}
$$

with the constraints

$$
D_{\mu} n^{a}=0, \quad n^{a} Y_{\mu}^{a}=0 .
$$

In [1] it was showed that the above decomposition of the gluon field is gauge invariant but not necessarily complete. In the weak coupling limit, $g \rightarrow 0$, a finite gluon field requires either $n=0$ or $\partial_{\mu} n=0$. If $n=0$, the gluon field is reduced to a vector field in the adjoint representation of $\mathrm{SU}(3)$ gauge group. For the other case, $\partial_{\mu} n=0$, the gluon is writen in terms of the vector fields $\hat{A}_{\mu}$ and $Y_{\mu}^{a}$ and includes the previous solution as a particular case. Accordingly, a field such that $n \neq 0$ or $\partial_{\mu} n \neq 0$ does not produce a finite gluon field in the weak coupling limit and, in this sense, can be viewed as a nonperturbative field. Among this class of fields, the simplest parametrisation for the covariant scalar field ${ }^{1}$ is $n^{a}=\delta^{a 1}(-\sin \theta)+\delta^{a 2}(\cos \theta)$. Then

$$
A_{\mu}^{a}=n^{a} \hat{A}_{\mu}+\delta^{a 3} \frac{1}{g} \partial_{\mu} \theta+\delta^{a 8} C_{\mu},
$$

where $C_{\mu}=Y_{\mu}^{8}$. The classical Lagrangian and equations of motion are independent of $\theta$ and are abelian like in $\hat{A}_{\mu}$ and $C_{\mu}$. Among the possible nonperturbative gluons given

\footnotetext{
${ }^{1}$ From the constraint equation $D n=0$ it follows that $n^{2}$ is constant. Our choice was $n^{2}=1$. A different value for the norm of $n$ is equivalent to a rescaling of $\hat{A}$.
} 
by (11), the simplest configuration has $\hat{A}=C=0$. The coupling to the fermionic fields requires only the Gell-Mann matrix $\lambda^{3}$, decoupling the different colour components. This suggests, naively, that such a field is able to produce either confining, non-confining or free particle solutions for the quarks.

The classical equations of motion are independent of $\theta$. However, a choice of a gauge condition, provides an equation for this field. For the Landau gauge, $\theta$ verifies a KleinGordon equation for a massless scalar field. Note that there is no boundary condition for $\theta$, i.e. the usual free particle solutions of the Klein-Gordon equation are not the only possible ones. Indeed, writing $\theta(t, \vec{r})=T(t) V(\vec{r})$, then

$$
\begin{gathered}
\frac{T^{\prime \prime}(t)}{T(t)}=\frac{\nabla^{2} V(\vec{r})}{V(\vec{r})}=\Lambda^{2}>0, \\
T(t)=a e^{\Lambda t}+b e^{-\Lambda t}, \\
V(\vec{r})=\sum_{l, m} V_{l}(r) Y_{l m}(\Omega), \\
V_{l}(r)=\frac{\alpha_{l}}{\sqrt{z}} I_{l+1 / 2}(z)+\frac{\beta_{l}}{\sqrt{z}} K_{l+1 / 2}(z),
\end{gathered}
$$

where $z=\Lambda r$ and $I_{l+1 / 2}(z)$ and $K_{l+1 / 2}(z)$ are modified spherical Bessel functions of the $1^{\text {st }}$ and $1^{\mathrm{rd}} \mathrm{kind}^{2}$. The lowest multipole solution is

$$
V_{0}(r)=A \frac{\sinh (\Lambda r)}{r}+B \frac{e^{-\Lambda r}}{r}
$$

and the associated gluon field is given by

$$
\begin{gathered}
A_{0}^{3}=\Lambda\left(e^{\Lambda t}-b e^{\Lambda t}\right) V_{0}(r), \\
\vec{A}_{0}^{3}=-\left(e^{\Lambda t}-b e^{\Lambda t}\right) \nabla V_{0}(r)
\end{gathered}
$$

From the lowest multipole solution one can derive a potential, which maybe suitable to describe heavy quarkonium. Indeed, assuming that quarks do not exchange energy, in the nonrelativistic approximation and leading order in $1 / m$, the spatial function in $A_{0}^{3}$, $V_{0}(r)$, can be viewed as a nonrelativistic potential ${ }^{3}$ and one can try to solve the associated Schrödinger equation. For the potential (8), the wave function goes to zero faster than an exponential for large quark distances,

$$
\psi(\vec{r})=\exp \left\{\frac{-2}{\Lambda} \sqrt{\frac{2 A}{m}} \exp \left(\frac{\Lambda r}{2}\right)\right\}
$$

As a first try to compute the heavy quarkonium spectra, we fixed $A, B$ and $\Lambda$ minimising the square of the difference between $V_{0}(r)+$ Constant and the Cornell potential [2] $V_{\text {Cornell }}=e / r+\sigma r(e=-0.25, \sqrt{\sigma}=427 \mathrm{MeV})$ integrated between $0.2 \mathrm{fm}$ and $1 \mathrm{fm}$.

\footnotetext{
2 Note that, by definition, the mass scale $\Lambda$ is independent of a rescaling of the gluon field.

3 The potential is $\sim 1 / r$ for short distances and goes to infinity for large quark distances.
} 
Charmonium

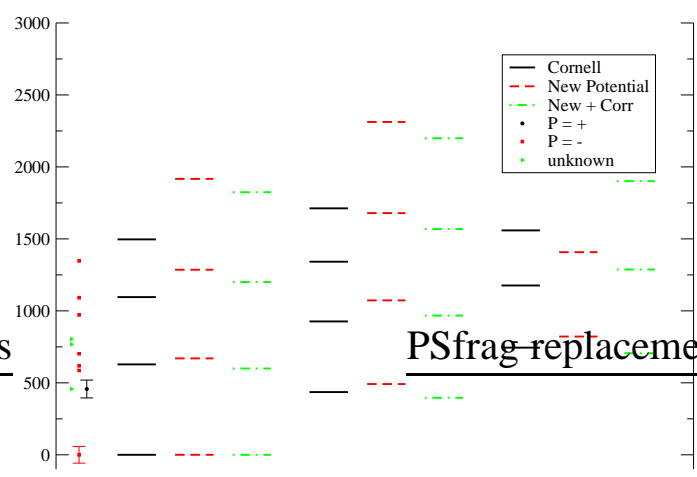

Bottomonium

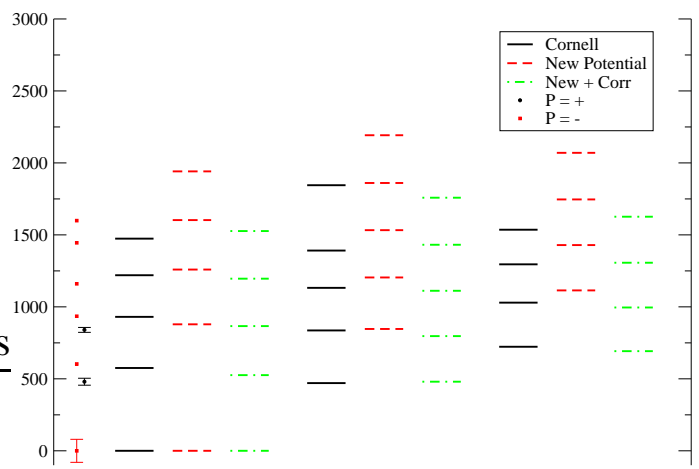

FIGURE 1. Heavy quarkonia spectra in MeV. The plots include the spin averaged experimental values.

This optimisation provides the following parameters $A=5.4, B=-1.0, \Lambda=281 \mathrm{MeV}$, Constant $=-1190 \mathrm{MeV}$; for these values $-24 \mathrm{MeV} \leq V_{\text {Cornell }}-\left(V_{0}+\right.$ Constant $) \leq 64$ $\mathrm{MeV}$ in the integration range considered. Then, we can compare the Schrödinger equation spectrum for the charmonium $\left(m_{c}=1.25 \mathrm{GeV}\right)$ and for the bottomonium $\left(m_{b}=4.25\right.$ $\mathrm{GeV}$ ) for the two potentials. The spectrum for the new potential shows an equal level spacing for both the charmonium and bottomonium spectra. If the $V_{0}$ charmonium spectrum is quite close to the Cornell spectrum, the botomonium shows clear deviations; see figure 1. The differences are the result of overestimating the strengh of $V_{0}(r)$ for smaller distances. Indeed, one can improve our potential linearising the full QCD equations around the above configuration. To lowest order, this is equivalent to add a term like $k / r$ to $V_{0}$. Computing $k$ perturbatively ${ }^{4}$ adjusting the $M[(1 P)]-M[(1 S)]$ bottomonium mass difference, gives $k=0.2448251$. The heavy quarkonia spectra, including this correction, is given in figure 1

In conclusion, classical configurations seem to be able to produce a spectra close to the Cornell potential. Hopefully, this is an indication that these configurations can be of help to understand strong interaction physics. Of course, there are a number of issues that need to be further investigated (definition of the potential parameters, inclusion of time dependence, decay rates). We are currently working on these topics and will provide a report soon.

\section{REFERENCES}

1. O.Oliveira, R. A. Coimbra, hep-ph/0305305

2. See G. S. Bali, Phys. Rep.343(2001)1-136|hep-ph/0001312| and references therein.

\footnotetext{
${ }^{4}$ For each stationary, the shift in energy due to this term is compatible with a perturbative treatment. Corrections are clearly below 10-20\%.
} 